\title{
Controller optimization with constraints on probabilistic peak responses
}

\author{
Ji-Hun Park $\dagger$ \\ MIDASIT Co., Ltd., Seoul, Korea \\ Kyung-Won Min $¥$ \\ Department of Architectural Engineering, Dankook University, Seoul, Korea \\ Hong-Gun Park $\ddagger$ \\ Department of Architecture, Seoul National University, Korea
}

(Received January 6, 2003, Accepted August 8, 2003)

\begin{abstract}
Peak response is a more suitable index than mean response in the light of structural safety. In this study, a controller optimization method is proposed to restrict peak responses of building structures subject to earthquake excitations, which are modeled as partially stationary stochastic process. The constraints are given with specified failure probabilities of peak responses. LQR is chosen to assure stability in numerical process of optimization. Optimization problem is formulated with weightings on controlled outputs as design variables and gradients of objective and constraint functions are derived. Full state feedback controllers designed by the proposed method satisfy various design objectives and output feedback controllers using LQG also yield similar results without significant performance deterioration.
\end{abstract}

Key words: stochastic process; crossing rate; failure probability; optimization; linear quadratic regulator.

\section{Introduction}

Many active structural control methods have been developed and implemented in the field of civil engineering to suppress excessive vibrations induced by earthquake or wind loads (Soong 1990, Housner et al. 1997). Due to the nature of such loads, their magnitudes and distributions cannot be exactly specified, but can be defined stochastically only. Therefore, the quantification of control effectiveness based on probabilistic concept is an important problem in controller design. Standard deviation is the most commonly used response quantity for stochastically excited structures, because most random disturbances can be approximated by white or filtered white noises and the response covariance matrix of a linear time invariant (LTI) system under a white noise excitation can be obtained by solving a Lyapunov equation. Further, the exact probability distribution of peak responses is not known (Lutes and Sarkani 1997). Accordingly, widely used control methodologies

$\uparrow$ Senior Developer

$\mp$ Professor 
such as Linear Quadratic Regulator (LQR) and Linear Quadratic Gaussian (LQG) control algorithms minimize the standard deviation of responses. However, in the light of structural safety, the critical response quantity is defined as a peak value rather than a standard deviation.

Spencer et al. (1994a) proposed a probabilistic controller design method for a single degree of freedom (SDF) structure considering the uncertainty in structural parameters. In their research, the probability of peak displacements exceeding critical value is used as an objective function and the root mean square (RMS) value of control forces is used as a constraint. May and Beck (1998) investigated an unconstrained optimization of the acceleration feedback controller for a three-story test building structure taking into account the parameter uncertainty in ground acceleration modeling. They minimized the failure probability defined by a safe region, but the level of the failure probability could not be specified. An important problem in the controller optimization is that a control gain may fall into an unstable region while seeking an optimal solution. In such cases, the probability distribution of the closed-loop system does not exist and the optimization process cannot be continued, since the closed-loop system responses diverge. In the previous two studies by Spencer et al. and May and Beck, there were no remarks on this issue. This is because the simple controller structures that they used make it easy to set up constraints on the stability.

There have been a lot of studies on controller optimization of which solution has the form of LQR controller. This is due to the facts that LQR controller has a simple stability condition expressed in terms of weightings on output variables, and that it can be easily extended to an output feedback controller with an appropriate observer, for example, LQG controller with Kalman filter. Studies on LQR or LQG controller optimization can be classified into three groups. First, Hsieh et al. (1989) and Zhu et al. (1997) studied the optimization with constraints on the covariance of output variables. Second, Zhu and Skelton (1991) and Rotea (1993) studied the optimization with constraints on $\mathrm{L}_{\infty}$ norm of output variables, which is deterministic norm and may be too conservative. Third, Toivonen and Mäkilä (1989) and Khargonekar and Rotea (1991) studied the optimization problem with multiple objective functions on RMS values of output variables. But none of them dealt with optimization with constraints on the probabilistic peak values of output variables considering the stochastic nature of external loads.

In this paper, a new controller optimization method is proposed to restrict the failure probabilities of peak responses below specified levels with minimum control force for multi degree of freedom (MDF) structures. The optimization problem is formulated with weightings on controlled responses as design variables. Gradients of objective function and inequality constraints are derived to make use of general gradient based optimization algorithms. The limit in the actuator stroke is also considered in the optimization problem. LQR controller with full state feedback is first chosen as a subject controller to deal with more complex and general controller types for MDF structures and to establish simple stability conditions. Next, the optimized full state feedback controller is extended to the output feedback controller using LQG with Kalman filters. The performance degradation due to the output feedback is evaluated in the numerical examples.

\section{Problem statements}

\subsection{Augmented design plant}

The augmented design plant is composed of a building structure, a control device and a 
disturbance frequency shaping filter. Each floor of the structure is assumed to have a diaphragm constraint so that the number of degree of freedom is equal to that of floors through static condensation and ignoring vertical deformations. The control device considered in this paper is a hybrid mass damper (HMD) that works as a tuned mass damper (TMD) for low level vibrations and as an active tuned mass damper (ATMD) for high level vibrations. The state space representation of the equation of motion for an $n$ degree of freedom structure with a HMD is

$$
\begin{gathered}
\dot{\boldsymbol{x}}_{s}=\boldsymbol{A}_{s} \boldsymbol{x}_{s}+\boldsymbol{B}_{s 1} f_{g}+\boldsymbol{B}_{s 2} u \\
\boldsymbol{y}_{s}=\boldsymbol{C}_{s 1} \boldsymbol{x}_{s}+\boldsymbol{D}_{s 1} u
\end{gathered}
$$

where

$$
\boldsymbol{x}_{s}=\left[\begin{array}{c}
\boldsymbol{q} \\
\dot{\boldsymbol{q}}
\end{array}\right] ; \quad \boldsymbol{y}_{s}=\left[\begin{array}{c}
\boldsymbol{q}_{d} \\
\ddot{\boldsymbol{q}}_{a b} \\
q_{d, H M D}
\end{array}\right]
$$

and $\boldsymbol{x}_{s}, \boldsymbol{y}_{s}, f_{g}$ and $u$ are, respectively, the $(2 n+2)$ state vector, the $(2 n+1)$ vector of controlled responses, the ground acceleration, and the control force, and $\boldsymbol{q}, \boldsymbol{q}_{d}, \ddot{\boldsymbol{q}}_{a b}$, and $q_{d, H M D}$ are, respectively, the $(n+1)$ vector of relative displacements of floors and the HMD, the $n$ inter-story drift vector, the $n$ absolute floor acceleration vector, and the HMD stroke. $\boldsymbol{A}_{s}, \boldsymbol{B}_{s 1}, \boldsymbol{B}_{s 2}, \boldsymbol{C}_{s 1}$, and $\boldsymbol{D}_{s 1}$ are the state matrix, the disturbance influence matrix, the control force influence matrix, the output matrix, and the matrix that represents the coupling between the control force and controlled responses, respectively.

The actuator dynamics is mathematically modeled with a first-order low pass filter (Yang et al. 1996) and represented by the following state space equation.

$$
\begin{gathered}
\dot{x}_{a}=-\omega_{a} x_{a}+\omega_{a} v \\
u=\alpha_{a} x_{a}
\end{gathered}
$$

where $v, x_{a}$, and $\omega_{a}$ are the control signal expressed as input voltage, the state of the actuator, the cut-off frequency, respectively, and $\alpha_{a}$ is a constant. The state space representation of Kanai-Tajimi filter is used for the frequency shaping filter of the ground acceleration.

$$
\begin{gathered}
\dot{\boldsymbol{x}}_{f}=\boldsymbol{A}_{f} \boldsymbol{x}_{f}+\boldsymbol{B}_{f} w \\
f_{g}=\boldsymbol{C}_{f} \boldsymbol{x}_{f}
\end{gathered}
$$

where

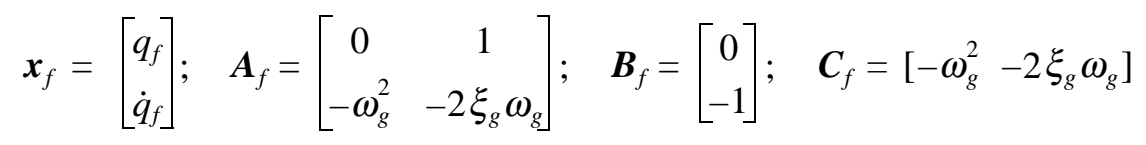


and $\boldsymbol{x}_{f}, w, q_{f}, \omega_{g}$, and $\xi_{g}$ are the state vector of the filter, the input white noise of the filter, the relative displacement of Kanai-Tajimi filter, the natural frequency of the filter, and the damping ratio of the filter, respectively. If soil property data is lack, a low pass filter, which is represented by the same state space equation form of Eqs. (6) and (7), can be used (Spencer et al. 1994b).

In sum, the augmented design plant for the structure with a HMD, the actuator, and the frequency shaping filter of ground acceleration is given as

$$
\begin{gathered}
\dot{\boldsymbol{x}}=\boldsymbol{A} \boldsymbol{x}+\boldsymbol{B}_{1} w+\boldsymbol{B}_{2} v \\
\boldsymbol{y}=\boldsymbol{C} \boldsymbol{x}+\boldsymbol{D} v
\end{gathered}
$$

where

$$
\begin{gathered}
\boldsymbol{x}=\left[\begin{array}{c}
\boldsymbol{x}_{s} \\
\boldsymbol{x}_{a} \\
\boldsymbol{x}_{f}
\end{array}\right] ; \quad \boldsymbol{A}=\left[\begin{array}{ccc}
\boldsymbol{A}_{s} & \boldsymbol{B}_{s 2} \alpha_{a} & \boldsymbol{B}_{s 1} \boldsymbol{C}_{f} \\
\boldsymbol{O} & \omega_{a} & \boldsymbol{O} \\
\boldsymbol{O} & \boldsymbol{O} & \boldsymbol{A}_{f}
\end{array}\right] ; \quad \boldsymbol{B}_{1}=\left[\begin{array}{c}
\boldsymbol{O} \\
\boldsymbol{O} \\
\boldsymbol{B}_{f}
\end{array}\right] ; \quad \boldsymbol{B}_{2}=\left[\begin{array}{c}
\boldsymbol{O} \\
\omega_{a} \\
\boldsymbol{O}
\end{array}\right] \\
\boldsymbol{y}=\left[\begin{array}{c}
\boldsymbol{y}_{s} \\
u \\
v
\end{array}\right] ; \quad \boldsymbol{C}=\left[\begin{array}{ccc}
\boldsymbol{C}_{s 1} & \boldsymbol{D}_{s 1} \alpha_{a} & \boldsymbol{O} \\
\boldsymbol{O} & \alpha_{a} & \boldsymbol{O} \\
\boldsymbol{O} & \boldsymbol{O} & \boldsymbol{O}
\end{array}\right] ; \quad \boldsymbol{D}=\left[\begin{array}{c}
\boldsymbol{O} \\
\boldsymbol{O} \\
1
\end{array}\right]
\end{gathered}
$$

where $\boldsymbol{x}$ is the $(2 n+5)$ state vector, $\boldsymbol{y}$ is the $(2 n+3)$ vector of output variables, and $\boldsymbol{O}$ and $\boldsymbol{O}$ are a zero matrix and a zero vector with appropriate dimensions. To represent the non-stationary property of earthquake, the envelope function proposed by Jennings et al. (1968) is multiplied to the white noise input, $w$, in the above augmented plant equations. A resulting sample ground acceleration and a scaled envelope function is presented in Fig. 1.

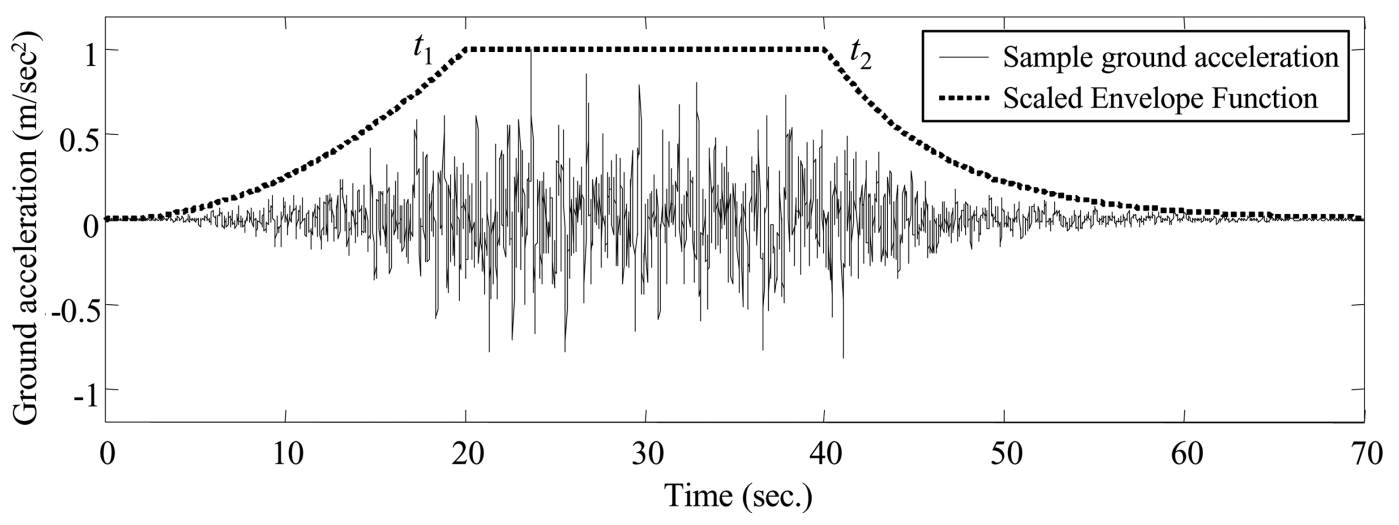

Fig. 1 Sample ground acceleration and the shape of envelope function 


\subsection{Definition of peak response}

From the viewpoint of safety and economy, it is required to specify the probability that the peak response exceeds prescribed critical value. Peak response can be expressed stochastically using a failure probability, which is the probability that the controlled response of the system exceeds the critical value. If the process $X$ is stationary and Gaussian and the failure probability at the initial time is zero, the failure probability of $X$ is defined as the probability that $|X|$ exceeds a prescribed barrier level $b$ during the interval, $t_{b}$, and is approximately given as (Lutes and Sarkani 1997)

$$
P\left(b, t_{b}\right)=1-\exp \left[-\eta_{|X|}^{+}(b) \cdot t_{b}\right]
$$

where $\eta_{|X|}^{+}(b)$ is the $X$ 's crossing rate. The crossing rate is defined as an average number of $|X|$ exceeding a prescribed level, $b$, during a unit time interval and expressed as

$$
\eta_{|X|}^{+}(b)=\frac{\sigma_{\dot{X}}}{\pi \sigma_{X}} \exp \left(\frac{-b^{2}}{2 \sigma_{X}^{2}}\right)
$$

where $\sigma_{X}$ and $\sigma_{\dot{X}}$ are, respectively, standard deviations of process $X$ and its time derivative (Lutes and Sarkani 1997).

Since the envelope function is flat for its largest amplitude part, the strong ground motion, presented in Fig. 1, can be assumed to be a stationary Gaussian process based on the assumption that the influence of the non-stationarity of the ground motion at the early rising stage is small and can be neglected. Since the steady-state output of a linear system subjected to the stationary Gaussian random excitation is also stationary and Gaussian, the plant output can be treated as a stationary and Gaussian process during the strong excitation interval for elastic structures and linear controllers. Accordingly, the above equations are applied for the remaining parts of this paper in spite of non-stationarity of earthquake and corresponding structural responses. Otherwise, a technique using evolutionary spectral density can be employed (Lin and Cai 1995), which is beyond the scope of this paper and reserved as a future research topic.

\subsection{Control algorithm}

Control algorithms can be classified into full state feedback control algorithms and output feedback control algorithms. The former employs the complete information of the plant state while the latter employs limited information. Therefore, the full state feedback control is more advantageous than the output feedback control. For practical reason, however, all state variables cannot be measured and an observer is often used to estimate state variables with limited number of measured responses. In this paper, only the optimization of the full state feedback controller is presented, but, if the state estimation error is negligible, an observer can be added based on the well-known separation principle (Burl 1999).

A full state feedback controller can be designed by optimizing the feedback control gain. However this process may cause instability of the closed loop system while seeking an optimum solution. In such case, the optimization cannot be continued since it becomes impossible to compute the norm of diverging responses. To overcome this drawback, the feedback gain needs to be restricted within the safe range that feedback gains stabilize the plant. But, for multi-variable systems, it is difficult to set up clear stability conditions involving the feedback gain itself. To solve such problem, this study 
adopts LQR because the positive semi-definite state weighting matrix and the positive definite control weighting matrix in its quadratic performance index always give stabilizing feedback gains.

For the output feedback control algorithm, LQG controller is investigated in this paper. In LQG, which is composed of LQR controller and Kalman filter, the plant disturbances and measurement noises are modeled by white noises. Further, plant disturbances and all measurement noises are assumed to be uncorrelated.

\section{Formulation of optimization problem}

\subsection{Optimization problem}

For the controller optimization, the variance of control force is selected as an objective function. That is, the optimization problem finds a control gain that requires a minimum control force in the mean sense. Constraints on controlled outputs - inter-story drifts, absolute floor accelerations, and HMD stroke - are given as specified levels of corresponding failure probabilities. The control force and control signal have no constraints, because if they are constrained, it may be impossible to find a controller satisfying constraints on structural responses. From optimization results, the peak control force and signal defined with specified failure probabilities can be calculated.

The nonlinear constrained optimization problem is defined as

$$
\begin{gathered}
\text { minimize } f(\boldsymbol{S})=V_{y_{2 n+2}} \\
\text { subject to } g_{k}(\boldsymbol{S})=1-\exp \left[-\eta_{\left|y_{k}\right|}^{+}\left(b_{k}\right) t_{s}\right]-P_{k} \leq 0, \quad k=1, \ldots, 2 n+1
\end{gathered}
$$

where $V_{y_{2 n+2}}$ is the variance of the control force that is the $(2 n+2)$-th output variable of Eq. (12) and $S$ is a $(2 n+3)$ by $(2 n+3)$ matrix containing design variables which are weightings in the performance index of LQR given by

$$
J=\int_{0}^{\infty} \boldsymbol{y}^{T} \boldsymbol{S} \boldsymbol{y} d t=\int_{0}^{\infty}\left(\boldsymbol{x}^{T} \boldsymbol{Q} \boldsymbol{x}+2 \boldsymbol{x}^{T} \boldsymbol{N} v+R v^{2}\right) d t
$$

in which

$$
\boldsymbol{Q}=\boldsymbol{C}^{T} \boldsymbol{S C} ; \quad \boldsymbol{N}=\boldsymbol{C}^{T} \boldsymbol{S D} ; \quad R=\boldsymbol{D}^{T} \boldsymbol{S D}
$$

In Eq. (16), $\eta_{\left|y_{k}\right|}^{+}\left(b_{k}\right)$ is the crossing rate of the $k$-th measured response, $y_{k}$, over the barrier level $b_{k}$, $t_{s}$ is the duration of stationary strong ground motion, and $P_{k}$ is the pre-specified failure probability for $y_{k}$.

In order to reduce the number of design variables to $2 n+3, S$ is assumed to have a diagonal matrix form as follows.

$$
S=\operatorname{diag}\left[s_{1}^{2}, s_{2}^{2}, \ldots, s_{2 n+3}^{2}\right]
$$

For the controller optimization, the weighting on the control signal, $s_{2 n+3}$, is set to be 1.0 and excluded from the design variables, since the LQR control gain depends on the relative values between weightings. 
The failure probability of each output variable is determined by the crossing rate over the duration of stationary process. Because there is one-to-one relationship between the crossing rate and the failure probability given by Eq. (13), the constraint Eq. (16) can be converted into the following nonlinear inequality equations.

$$
g_{k}(S)=\frac{\sqrt{V_{\dot{y}_{k}}}}{\pi \sqrt{V_{y_{k}}} \exp \left(\frac{b_{k}^{2}}{2 V_{y_{k}}}\right)}-\rho_{k} \leq 0, \quad k=1, \ldots, 2 n+1
$$

where $V_{y_{k}}$ and $V_{\dot{y}_{k}}$ are variances of $y_{k}$ and $\dot{y}_{k}$, respectively, and $\rho_{k}$ is an upper bound of crossing rate calculated from the $P_{k}$ of Eq. (16) and the basic relationship of Eq. (13).

The optimal control gain, $\boldsymbol{G}$, of $\mathrm{LQR}$ for a linear system under a Gaussian white noise disturbance is known to be independent of the disturbance (Burl 1999) and given by

$$
\boldsymbol{G}=R^{-1}\left(\boldsymbol{B}_{2}^{T} \boldsymbol{P}+N^{T}\right)
$$

where $\boldsymbol{P}$ is the Riccati matrix, which can be calculated from the algebraic Ricatti equation (Anderson and Moore 1989)

$$
\boldsymbol{P A}+\boldsymbol{A}^{T} \boldsymbol{P}-\left(\boldsymbol{P} \boldsymbol{B}_{2}+N\right) R^{-1}\left(\boldsymbol{P} \boldsymbol{B}_{2}+N\right)^{T}+\boldsymbol{Q}=\boldsymbol{O}
$$

In order to calculate the objective function and constraints, the covariance matrices of $\boldsymbol{y}$ and $\dot{\boldsymbol{y}}$ need to be obtained. The covariance matrix of the output vector $\boldsymbol{y}$ is calculated from a Lyapunov equation of the closed loop system (Burl 1999), and given as

$$
\Sigma_{y y}=C_{c} \Sigma_{x x} C_{c}^{T}
$$

subject to

$$
\boldsymbol{A}_{c} \boldsymbol{\Sigma}_{x x}+\Sigma_{x x} \boldsymbol{A}_{c}^{T}+2 \pi S_{w} \boldsymbol{B}_{1} \boldsymbol{B}_{1}^{T}=\boldsymbol{O}
$$

where

$$
\boldsymbol{A}_{c}=\boldsymbol{A}-\boldsymbol{B}_{2} \boldsymbol{G} ; \quad \boldsymbol{C}_{c}=\boldsymbol{C}-\boldsymbol{D} \boldsymbol{G}
$$

and $\boldsymbol{\Sigma}_{x \boldsymbol{x}}$ and $\boldsymbol{\Sigma}_{y \boldsymbol{y}}$ are, respectively, the covariance matrices of state vector $\boldsymbol{x}$ and output vector $\boldsymbol{y}$, and $S_{w}$ is the power spectral density (PSD) of the white noise disturbance.

The derivative of the output vector $\boldsymbol{y}$ can be written by

$$
\dot{\boldsymbol{y}}=\boldsymbol{C}_{c} \dot{\boldsymbol{x}}=\boldsymbol{C}_{c} \boldsymbol{A}_{c} \boldsymbol{x}+\boldsymbol{C}_{c} \boldsymbol{B}_{1} w
$$

It can be seen in Eq. (26) that $\dot{\boldsymbol{y}}$ is a linear combination of the state vector and the white noise disturbance. Since the state of LTI system under a white noise input is the Markov process, the state vector $\boldsymbol{x}$ and the white noise disturbance $w$ in Eq. (26) are independent each other. Consequently, 
the covariance matrix of $\dot{\boldsymbol{y}}$ is obtained as

$$
\boldsymbol{\Sigma}_{\dot{y} \dot{y}}=\boldsymbol{C}_{c}\left[\boldsymbol{A}_{c} \boldsymbol{\Sigma}_{x x} \boldsymbol{A}_{c}^{T}+2 \pi S_{w} \delta(0) \boldsymbol{B}_{1} \boldsymbol{B}_{1}^{T}\right] \boldsymbol{C}_{c}^{T}
$$

where $\delta(0)$ is a Dirac delta function having an infinite value at zero. To avoid an infinite covariance matrix, the white noise disturbance is assumed to be band-limited. This can be justified because, for real implementation of control problem, all signals are discretely represented with a sampling time, and frequency contents of those discrete signals higher than the Nyquist frequency are filtered out. In this study, the finite covariance matrix of $\dot{\boldsymbol{y}}$ is calculated using a basic conversion rule (Burl 1999) between the variance of a continuous signal and its discrete counterpart as

$$
\boldsymbol{\Sigma}_{\dot{y} \dot{y}}=\boldsymbol{C}_{c}\left[\boldsymbol{A}_{c} \boldsymbol{\Sigma}_{x \boldsymbol{x}} \boldsymbol{A}_{c}^{T}+\frac{2 \pi S_{w}}{\Delta t} \boldsymbol{B}_{1} \boldsymbol{B}_{1}^{T}\right] \boldsymbol{C}_{c}^{T}
$$

where $\Delta t$ is the sampling time.

\subsection{Gradients of objective function and inequality constraints}

Algorithms to solve a nonlinear constrained optimization problem are grouped into gradient based methods and direct search methods. For the problem with large number of design variables, the former is known to be more effective than the latter if the gradients of objective function and constraints are continuous (Belegundu and Chandrupatla 1999). Accordingly, a gradient based method is adopted in this paper, and the gradient calculations necessary in the optimization procedure are derived in this section.

The gradients of the objective function and the $k$-th constraint with respect to the $p$-th design variable $s_{p}$ in Eq. (19) are obtained using chain rule such that

$$
\begin{gathered}
\frac{\partial f(\cdot)}{\partial s_{p}}=\frac{\partial V_{y_{2 n+2}}}{\partial s_{p}} \\
\frac{\partial g_{k}(\cdot)}{\partial s_{p}}=\frac{\partial g_{k}(\cdot)}{\partial V_{y_{k}}} \frac{\partial V_{y_{k}}}{\partial s_{p}}+\frac{\partial g_{k}(\cdot)}{\partial V_{\dot{y}_{k}}} \frac{\partial V_{\dot{y}_{k}}}{\partial s_{p}}, \quad k=1, \ldots, 2 n+1
\end{gathered}
$$

The derivative of the $k$-th constraint $g_{k}(\cdot)$ with respect to the corresponding variances $V_{y_{k}}$ and $V_{\dot{y}_{k}}$ in the above equation can be obtained by simple differentiation. The derivatives of $V_{y_{k}}$ and $V_{\dot{y}_{k}}$ with respect to the $p$-th design variable $s_{p}$ can be obtained using the elements of Riccati matrix $\boldsymbol{P}$ and applying the chain rule as

$$
\begin{gathered}
\frac{\partial V_{y_{k}}}{\partial s_{p}}=\sum_{i=1}^{2 n+5} \sum_{j=1}^{2 n+5} \frac{\partial V_{y_{k}}}{\partial \boldsymbol{P}_{i j}} \frac{\partial \boldsymbol{P}_{i j}}{\partial s_{p}}=\sum_{i=1}^{2 n+5} \sum_{j=1}^{2 n+5}\left[\frac{\partial \boldsymbol{\Sigma}_{y \boldsymbol{y}}}{\partial \boldsymbol{P}_{i j}}\right]_{k k}\left[\frac{\partial \boldsymbol{P}}{\partial s_{p}}\right]_{i j} \quad k=1, \ldots, 2 n+1 \\
\frac{\partial V_{\dot{y}_{k}}}{\partial s_{p}}=\sum_{i=1}^{2 n+5} \sum_{j=1}^{2 n+5} \frac{\partial V_{\dot{y}_{k}}}{\partial \boldsymbol{P}_{i j}} \frac{\partial \boldsymbol{P}_{i j}}{\partial s_{p}}=\sum_{i=1}^{2 n+5} \sum_{j=1}^{2 n+5}\left[\frac{\partial \boldsymbol{\Sigma}_{i \dot{y}}}{\partial \boldsymbol{P}_{i j}}\right]_{k k}\left[\frac{\partial \boldsymbol{P}}{\partial s_{p}}\right]_{i j} \quad k=1, \ldots, 2 n+1
\end{gathered}
$$

where $\boldsymbol{P}_{i j}$ is the $(i, j)$-th component of $\boldsymbol{P}$. 
The derivatives of the covariance matrices of $\boldsymbol{y}$ and $\dot{\boldsymbol{y}}$, with respect to the component $\boldsymbol{P}_{i j}$ in Eqs. (31) and (32) are obtained by partially differentiating Eqs. (23) and (28) with respect to $\boldsymbol{P}_{i j}$ as

$$
\begin{gathered}
\frac{\partial \boldsymbol{\Sigma}_{y y}}{\partial \boldsymbol{P}_{i j}}=\frac{\partial \boldsymbol{C}_{c}}{\partial \boldsymbol{P}_{i j}} \boldsymbol{\Sigma}_{x x} \boldsymbol{C}_{c}^{T}+\boldsymbol{C}_{c} \frac{\partial \boldsymbol{\Sigma}_{x x}}{\partial \boldsymbol{P}_{i j}} \boldsymbol{C}_{c}^{T}+\boldsymbol{C}_{c} \boldsymbol{\Sigma}_{\boldsymbol{x} x} \frac{\partial \boldsymbol{C}_{c}^{T}}{\partial \boldsymbol{P}_{i j}} \\
\frac{\partial \boldsymbol{\Sigma}_{j \dot{y}}}{\partial \boldsymbol{P}_{i j}}=\frac{\partial \boldsymbol{C}_{c}}{\partial \boldsymbol{P}_{i j}}\left(\boldsymbol{A}_{c} \boldsymbol{\Sigma}_{x x} \boldsymbol{A}_{c}^{T}+\frac{2 \pi S_{w}}{\Delta t} \boldsymbol{B}_{1} \boldsymbol{B}_{1}\right) \boldsymbol{C}_{c}^{T} \\
+\boldsymbol{C}_{c}\left(\frac{\partial \boldsymbol{A}_{c}}{\partial \boldsymbol{P}_{i j}} \boldsymbol{\Sigma}_{x \boldsymbol{x}} \boldsymbol{A}_{c}^{T}+\boldsymbol{A}_{c} \frac{\partial \boldsymbol{\Sigma}_{x x}}{\partial \boldsymbol{P}_{i j}} \boldsymbol{A}_{c}^{T}+\boldsymbol{A}_{c} \boldsymbol{\Sigma}_{x x} \frac{\partial \boldsymbol{A}_{c}^{T}}{\partial \boldsymbol{P}_{i j}}\right) \boldsymbol{C}_{c}^{T} \\
+\boldsymbol{C}_{c}\left(\boldsymbol{A}_{c} \boldsymbol{\Sigma}_{x x} \boldsymbol{A}_{c}^{T}+\frac{2 \pi S_{w}}{\Delta t} \boldsymbol{B}_{1} \boldsymbol{B}_{1}\right) \frac{\partial \boldsymbol{C}_{c}^{T}}{\partial \boldsymbol{P}_{i j}}
\end{gathered}
$$

The derivatives of the matrices $\boldsymbol{A}_{c}$ and $\boldsymbol{C}_{c}$ with respect to the component $\boldsymbol{P}_{i j}$ in Eqs. (33) and (34) are calculated as

$$
\begin{aligned}
& \frac{\partial \boldsymbol{A}_{c}}{\partial \boldsymbol{P}_{i j}}=-\boldsymbol{B}_{2} R^{-1} \boldsymbol{B}_{2}^{T} \frac{\partial \boldsymbol{P}}{\partial \boldsymbol{P}_{i j}} \\
& \frac{\partial \boldsymbol{C}_{c}}{\partial \boldsymbol{P}_{i j}}=-\boldsymbol{D} R^{-1} \boldsymbol{B}_{2}^{T} \frac{\partial \boldsymbol{P}}{\partial \boldsymbol{P}_{i j}}
\end{aligned}
$$

where

$$
\left[\frac{\partial \boldsymbol{P}}{\partial \boldsymbol{P}_{i j}}\right]_{k l}= \begin{cases}1 & \text { if } \quad(k=i \text { and } l=j) \\ 0 & \text { otherwise }\end{cases}
$$

The derivative of the covariance of the state vector with respect to the Riccati matrix, $\partial \boldsymbol{\Sigma}_{x x} / \partial \boldsymbol{P}_{i j}$, in Eqs. (33) and (34) is obtained by solving the following Lyapunov equation, which is derived by differentiating the Lyapunov equation (24) with respect to $\boldsymbol{P}_{i j}$.

$$
\boldsymbol{A}_{c} \frac{\partial \boldsymbol{\Sigma}_{x x}}{\partial \boldsymbol{P}_{i j}}+\frac{\partial \boldsymbol{\Sigma}_{x x}}{\partial \boldsymbol{P}_{i j}} \boldsymbol{A}_{c}^{T}+\frac{\partial \boldsymbol{A}_{c}}{\partial \boldsymbol{P}_{i j}} \boldsymbol{\Sigma}_{x x}+\boldsymbol{\Sigma}_{x x} \frac{\partial \boldsymbol{A}_{c}^{T}}{\partial \boldsymbol{P}_{i j}}=\mathbf{0}
$$

The derivative of the Riccati matrix with respect to the $p$-th component of the weighting matrix $s_{p}$, $\partial \boldsymbol{P} / \partial s_{p}$, is obtained by solving the following Lyapunov equation, which is obtained by differentiating the Riccati Eq. (22) with respect to $s_{p}$

$$
\frac{\partial \boldsymbol{P}}{\partial s_{p}} \boldsymbol{A}_{c}+\boldsymbol{A}_{c}^{T} \frac{\partial \boldsymbol{P}}{\partial s_{p}}+(\boldsymbol{D} \boldsymbol{G}-\boldsymbol{C})^{T} \frac{\partial \boldsymbol{S}}{\partial s_{p}}(\boldsymbol{D} \boldsymbol{G}-\boldsymbol{C})=\mathbf{0}
$$

where

$$
\left[\frac{\partial S}{\partial s_{p}}\right]_{i j}= \begin{cases}2 s_{p} & \text { if }(i=j=p) \\ 0 & \text { otherwise }\end{cases}
$$

Since the state space equation of the actuator is strictly proper and its number of state is one as shown in Eq. (4), only one component is non-zero in the matrix $\boldsymbol{B}_{2}$ of Eq. (11). In this case, 
$\partial \boldsymbol{A}_{c} / \partial \boldsymbol{P}_{i j}$ in Eqs. (35) and $\partial \boldsymbol{C}_{c} / \partial \boldsymbol{P}_{i j}$ in Eq. (36) are non-zero matrix only for $i=2 n+3$ and $j=1$, $2, \ldots, 2 n+5$. Therefore, only $2 n+5$ of both $\partial \boldsymbol{\Sigma}_{x x} / \partial \boldsymbol{P}_{i j}$ 's in Eq. (33) and $\partial \boldsymbol{\Sigma}_{j \dot{y}} / \partial \boldsymbol{P}_{i j}$ 's in Eq. (34) need to be calculated and the rest are zero. Further, Eqs. (35) and (36) are calculated only once during the entire optimization process because $R$ is a fixed weighting for the control signal.

\section{Numerical analysis}

A ten-story shear building shown in Fig. 2 is used for the numerical analysis using the proposed optimization process. The order of the design plant is 25 . The larger the DOF of the structure becomes, the more difficult convergence is. But, in many cases, this may not be a problem since most civil structural vibration is dominated by only a few modes and the design plants identified from experiments have small order.

Structural properties are presented in Table 1. The HMD installed on the top floor consists of a TMD and a hydraulic actuator. The mass of the TMD is $0.5 \%$ of the total mass of the building. The natural frequency and damping ratio of the TMD are determined from the equation proposed by Ayorinde (Ayorinde and Warburton 1980) for optimal passive control.

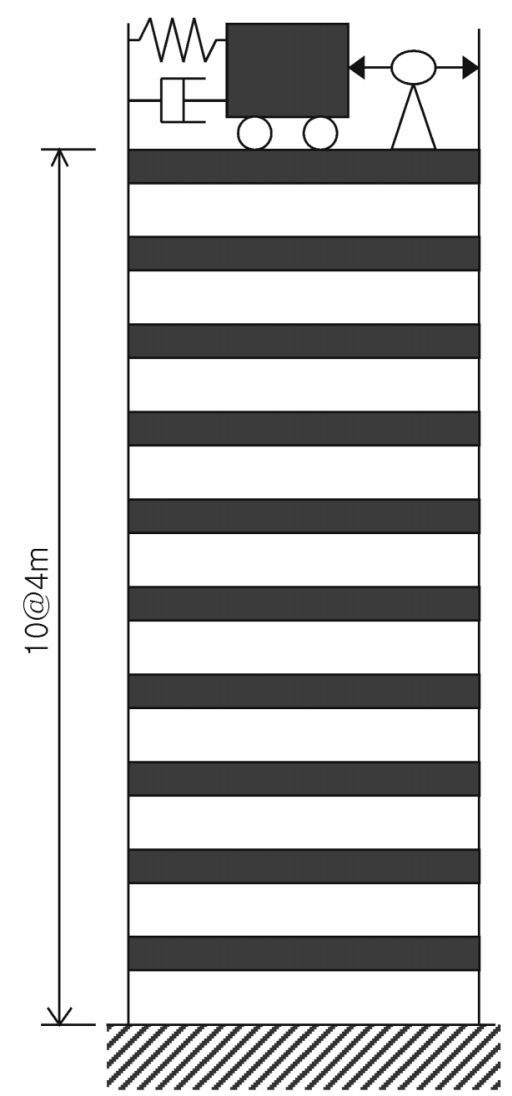

Fig. 2 Example ten-story shear building with a hybrid mass damper 
Table 1 Structural parameters

\begin{tabular}{ccccc}
\hline \hline Floor & Mass $(\mathrm{kg})$ & Stiffness $(\mathrm{kgf} / \mathrm{cm})$ & Natural frequencies $(\mathrm{Hz})$ & Damping ratio \\
\hline 1 & 24300 & 10000 & 0.42 & 0.02 \\
$2,3,4$ & 24300 & 8000 & $1.16 / 1.90 / 2.64$ & for all structural \\
$5,6,7$ & 24300 & 7000 & $3.22 / 3.82 / 4.20$ & modes \\
$8,9,10$ & 24300 & 5000 & $4.67 / 5.09 / 5.51$ & \\
\hline
\end{tabular}

Table 2 Parameters of ground acceleration model and actuator

\begin{tabular}{ccc}
\hline \hline & Mean peak ground acceleration & $0.1 \mathrm{~g}$ \\
Ground & PSD of white noise input & $7.949 \times 10^{-4} \mathrm{~m}^{2} / \mathrm{sec}^{3}$ \\
acceleration & Natural frequency of filter & $15.6 \mathrm{rad} / \mathrm{sec}$ \\
& Damping ratio of filter & 0.6 \\
& Envelope function & $t_{1}=20 \mathrm{sec}, t_{2}=40 \mathrm{sec}, \mathrm{c}=0.15$ \\
\hline \multirow{2}{*}{ Actuator } & $\omega_{a}$ & $11.94 \mathrm{rad} / \mathrm{sec}$ \\
& $\alpha_{a}$ & 400 \\
\hline
\end{tabular}

Table 3 Constraint sets on peak responses

\begin{tabular}{cccc}
\hline \hline Constraint set & $\mathrm{A}$ & $\mathrm{B}$ & $\mathrm{C}$ \\
\hline Inter-story drift & $2 \mathrm{~cm}$ & $2 \mathrm{~cm}$ & $2 \mathrm{~cm}$ \\
Floor absolute acceleration & $0.2 \mathrm{~g}$ & $0.2 \mathrm{~g}$ & $0.2 \mathrm{~g}$ \\
HMD stroke & $1.5 \mathrm{~m}$ & $1.75 \mathrm{~m}$ & $2 \mathrm{~m}$ \\
\hline
\end{tabular}

Parameters used for the Kanai-Tajimi filter, the envelope function of the filter input, and the actuator model are presented in Table 2. The PSD of the white noise input, $S_{w}$, for the Kanai-Tajimi filter is determined to set the mean peak ground acceleration to $0.1 \mathrm{~g}$ from 1000 samples. Parameters of the envelope function are selected such that the strong motion continues stationarily from $t_{1}$ to $t_{2}$ with falloff parameter $c$ (Jennings et al. 1968).

Design variables are given by

$$
\boldsymbol{S}=\operatorname{diag}\left[s_{d, 1}^{2}, \ldots, s_{d, 10}^{2}, s_{a, 1}^{2}, \ldots, s_{a, 10}^{2}, s_{m}^{2}, s_{u}^{2}, 1\right]
$$

where diagonal elements represents weightings on measured responses, which correspond to ten inter-story drifts, ten absolute floor accelerations, an HMD stroke, a control force, and a control signal, respectively. As previously mentioned, the weighting on the control signal is set to be 1 . In order to avoid an ill-conditioning and improve the optimization performance, scale factors of $100^{2}$, $1,1,0.01^{2}, 10^{2}$ are multiplied to five groups of weightings in Eq. (41), because they have different dimensions.

Three different constraints sets on inter-story drifts, absolute floor accelerations, and an HMD stroke are given in Table 3. They are classified as $\mathrm{A}, \mathrm{B}$, and $\mathrm{C}$ according to the level of the HMD stroke. The optimization is carried out using the sequential quadratic programming algorithm in MATLAB Optimization Tool Box (Coleman et al. 1999). All peak responses including the control force and control signal presented in the remaining parts of this paper are based on $5 \%$ failure 
probability assuming that the duration of the stationary large response is the same as that of the stationary strong ground motion, i.e. from 20 to $40 \mathrm{sec}$.

\subsection{Full state feedback controller design}

The controller optimization is performed for two different cases and the results are presented in Table 4 with activated constraints shaded. In the first case, denoted as LQR(I), $s_{d, i}(i=1$ to 10$), s_{a, i}$ ( $i=1$ to 10$), s_{m}$ and $s_{u}$ in Eq. (41) are included in the LQR performance index. In the second case, denoted as LQR(II), only $s_{d, 2}, s_{a, 10}, s_{m}$ and $s_{u}$ are included, because peak inter-story drift and absolute floor acceleration occur in the second and tenth floor, respectively, without control. Initial values for $s_{d, i}(i=1$ to 10$), s_{a, i}(i=1$ to 10$), s_{m}$ and $s_{u}$ are 100,100, 100 and 1 , respectively, for both cases. Constraints on all of the inter-story drifts, the absolute floor accelerations, and the HMD stroke are imposed for both cases.

The constraints on controlled responses except the second inter-story drift and the tenth floor absolute acceleration are not activated so that they are not presented in Table 4. It is observed that the peak responses and control force for $L Q R(I)$ and $L Q R(I I)$ are not significantly different in spite of the difference in the number of design variables. Additionally, LQR(II), where the smaller number of design variables is used, converges faster than LQR(I). Consequently, only LQR(II) with five design variables will be discussed hereafter.

Table 4 indicates that a strict stroke constraint requires large control force. This is because the HMD is tuned to the first structural mode and limiting its stroke implies decreasing control effect on that mode. When the stroke limit is not large enough for the first mode control, the control efforts move onto higher modes to compensate consequent increase in overall responses. This requires large control force since higher natural frequencies require the faster HMD movement. Fig. 3 shows the seesaw relation between responses of the first and second mode. That is, the LQR(II) with constraint $\mathrm{B}$ (stroke $=1.75 \mathrm{~m}$ ) is superior to LQR(II) with constraint A (stroke $=1.5 \mathrm{~m}$ ) in control of the first mode, while the latter with a smaller stroke limit is superior in control of the second mode. For the optimization with a stroke constraint of $1.25 \mathrm{~m}$, not presented in Table 4, no feasible solution is found. This means that there exists a lower limit of the stroke, which can be determined from optimizations with different stroke constraints.

Table 4 Controlled peak responses (Activated constraints are shaded)

\begin{tabular}{ccccccc}
\hline \hline \multirow{2}{*}{ Controller } & Constraint & $\begin{array}{c}2^{\text {nd }} \\
\text { inter-story } \\
\text { Drift }(\mathrm{cm})\end{array}$ & $\begin{array}{c}10^{\text {th }} \text { floor absolute } \\
\text { acceleration } \\
(\mathrm{g})\end{array}$ & $\begin{array}{c}\text { Stroke } \\
(\mathrm{m})\end{array}$ & $\begin{array}{c}\text { Control } \\
\text { force } \\
(\mathrm{kN})\end{array}$ & $\begin{array}{c}\text { Control } \\
\text { signal } \\
(\mathrm{V})\end{array}$ \\
\hline \multicolumn{2}{c}{ No control } & 3.67 & 0.30 & $\cdot$ & $\cdot$ & $\cdot$ \\
\hline \multicolumn{2}{c}{ Passive control } & 2.70 & 0.26 & 1.41 & $\cdot$ & $\cdot$ \\
\hline \multirow{2}{*}{ LQR (I) } & $\mathrm{A}$ & 2.00 & 0.18 & 1.50 & 10.74 & 37.4 \\
& $\mathrm{~B}$ & 2.00 & 0.20 & 1.75 & 5.79 & 18.0 \\
\hline \multirow{2}{*}{ LQR (II) } & $\mathrm{C}$ & 2.00 & 0.20 & 1.92 & 5.69 & 17.8 \\
& $\mathrm{~A}$ & 2.00 & 0.18 & 1.50 & 10.65 & 34.6 \\
& $\mathrm{~B}$ & 2.00 & 0.20 & 1.75 & 5.84 & 17.4 \\
\hline
\end{tabular}




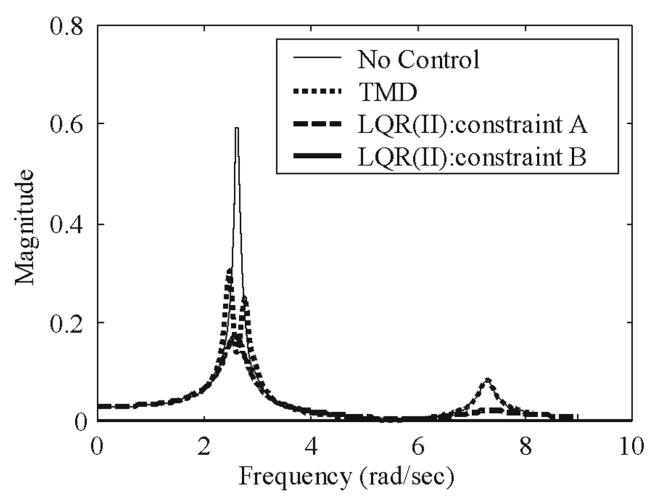

(a)

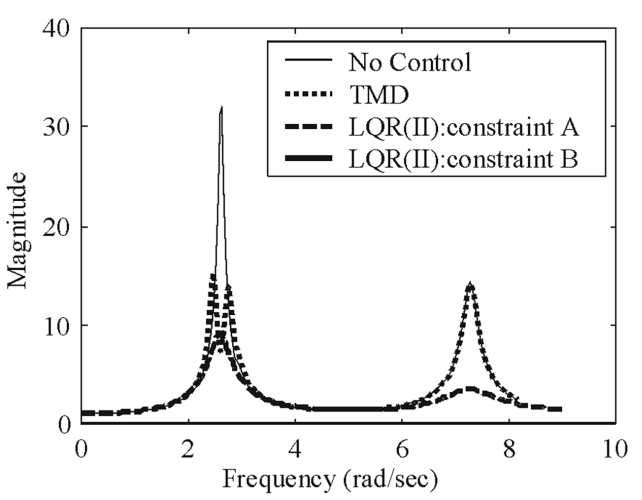

(b)

Fig. 3 Transfer functions for different constraints on stroke limit: (a) transfer function from the ground acceleration filter input to the 2nd inter-story drift, (b) transfer function from the ground acceleration filter input to the 10th floor absolute acceleration

To investigate the influence of the actuator cut-off frequency, the proposed optimization process is carried out for four different cut-off frequencies corresponding to the first to fourth natural frequencies, $\omega_{1}$ to $\omega_{4}$, of the structure. Table 5 , where peak control forces and signals corresponding to different cut-off frequencies are presented, shows that, as the cut-off frequency increases, peak control signals decrease but peak control forces change little. Since cut-off frequency is recommended to be as small as possible to prevent the spill-over effect due to noises and the uncertainty in the higher modes of the structure, optimization results for various cut-off frequencies will help to choose or produce an actuator and signal processing equipment.

To investigate the effect of initial weightings on optimization results, four different sets of initial weightings, denoted as $\mathrm{W}(\mathrm{I})$ to $\mathrm{W}(\mathrm{IV})$ in Table 6 , are examined. In this table, peak control forces and signals correspond to those of LQR with initial weightings. Table 7 shows that final weightings obtained from the optimization are very different for different initial values. However, the peak control force and signal for each constraint set have similar values except a few cases of W(II) for constraint set $\mathrm{A}$ and $\mathrm{W}(\mathrm{I})$ for constraint set $\mathrm{B}$ and $\mathrm{C}$. Also, activated constraints are same for different initial weighting sets, excluding those exceptional cases. These observations illustrates that the proposed optimization method gives reasonable solution by adjusting proportion of weightings regardless of their absolute values.

Table 5 Peak control forces and signals corresponding to different cut-off frequencies

\begin{tabular}{cccccc}
\hline \hline \multirow{2}{*}{ Constraint } & \multirow{2}{*}{ Control effort } & \multicolumn{4}{c}{ Cut-off frequency } \\
\cline { 3 - 6 } & & $\omega_{1}$ & $\omega_{2}$ & $\omega_{3}$ & $\omega_{4}$ \\
\hline \multirow{2}{*}{$\mathrm{A}$} & Control force $(\mathrm{kN})$ & 11.92 & 11.92 & 10.65 & 10.60 \\
& Control signal (V) & 1120.8 & 407.0 & 34.6 & 31.7 \\
\hline \multirow{2}{*}{$\mathrm{B}$} & Control force $(\mathrm{kN})$ & 5.85 & 5.82 & 5.84 & 5.80 \\
& Control signal (V) & 44.6 & 26.5 & 17.4 & 16.2 \\
\hline \multirow{2}{*}{$\mathrm{C}$} & Control force (kN) & 5.62 & 5.62 & 5.62 & 5.62 \\
& Control signal (V) & 48.6 & 23.0 & 16.1 & 16.1 \\
\hline
\end{tabular}


Table 6 Initial weighting sets

\begin{tabular}{ccccccc}
\hline \hline $\begin{array}{c}\text { Initial } \\
\text { weighting }\end{array}$ & $s_{d, 2}$ & $s_{a, 10}$ & $s_{m}$ & $s_{u}$ & $\begin{array}{c}\text { Peak } \\
\text { control force } \\
(\mathrm{kN})\end{array}$ & $\begin{array}{c}\text { Peak } \\
\text { control signal } \\
(\mathrm{V})\end{array}$ \\
\hline $\mathrm{W}(\mathrm{I})$ & 1 & 1 & 1 & 1 & 0.00 & 0.01 \\
$\mathrm{~W}(\mathrm{II})$ & 10 & 10 & 10 & 1 & 0.20 & 0.51 \\
W(III) & 100 & 100 & 100 & 1 & 3.28 & 8.99 \\
$\mathrm{~W}(\mathrm{IV})$ & 1000 & 1000 & 1000 & 1 & 22.58 & 72.68 \\
\hline
\end{tabular}

Table 7 Final weightings for different initial weighting sets

\begin{tabular}{|c|c|c|c|c|c|c|c|c|}
\hline \multirow{2}{*}{ Constraint } & \multirow{2}{*}{$\begin{array}{c}\text { Initial } \\
\text { weighting }\end{array}$} & \multicolumn{5}{|c|}{ Final weightings } & \multirow{2}{*}{$\begin{array}{c}\text { Peak } \\
\text { control force } \\
(\mathrm{kN})\end{array}$} & \multirow{2}{*}{$\begin{array}{c}\text { Peak } \\
\text { control signal } \\
(\mathrm{V})\end{array}$} \\
\hline & & $s_{d, 2}$ & $s_{a, 10}$ & $s_{m}$ & $s_{u}$ & $s_{\text {иа }}$ & & \\
\hline \multirow{4}{*}{ A } & $\mathrm{W}(\mathrm{I})$ & 518.78 & 253.37 & 456.13 & 0.00 & 1.00 & 10.95 & 32.75 \\
\hline & $\mathrm{W}(\mathrm{II})$ & 196.67 & 454.99 & 259.97 & 0.37 & 1.00 & 13.48 & 41.09 \\
\hline & W(III) & 712.91 & 172.78 & 616.93 & 0.00 & 1.00 & 10.65 & 34.60 \\
\hline & $\mathrm{W}(\mathrm{IV})$ & 1691.20 & 280.98 & 1445.30 & 6.96 & 1.00 & 10.59 & 35.10 \\
\hline \multirow{4}{*}{ B } & $\mathrm{W}(\mathrm{I})$ & 436.15 & 0.00 & 308.37 & 0.00 & 1.00 & 8.06 & 22.87 \\
\hline & W(II) & 1629.20 & 1677.20 & 885.49 & 33.47 & 1.00 & 5.82 & 18.56 \\
\hline & W(III) & 92.76 & 182.12 & 63.13 & 0.00 & 1.00 & 5.84 & 17.42 \\
\hline & $\mathrm{W}(\mathrm{IV})$ & 1106.60 & 1142.30 & 602.01 & 22.67 & 1.00 & 5.81 & 17.96 \\
\hline \multirow{4}{*}{$\mathrm{C}$} & $\mathrm{W}(\mathrm{I})$ & 436.15 & 0.00 & 308.37 & 0.00 & 1.00 & 8.06 & 22.87 \\
\hline & W(II) & 1382.80 & 2267.10 & 9.41 & 43.54 & 1.00 & 5.64 & 17.84 \\
\hline & W(III) & 224.28 & 393.67 & 0.00 & 6.87 & 1.00 & 5.62 & 16.08 \\
\hline & $\mathrm{W}(\mathrm{IV})$ & 956.82 & 1572.10 & 0.00 & 30.11 & 1.00 & 5.63 & 17.33 \\
\hline
\end{tabular}

\subsection{Output feedback controller design}

This section presents extension of LQR controller optimized in the previous section to LQG controllers by adding Kalman filters. Since it is hard to measure all states of plant, output feedback control is required in most civil structures. In this example, the Kanai-Tajimi filter input is used as a plant disturbance. Measured responses are assumed to be absolute accelerations of the second, sixth, and tenth floors and the HMD. PSD of all measurement noises is assumed to be 5\% of the variance of the tenth floor absolute acceleration when the HMD acts passively.

Design results of full order LQG controllers are presented in Table 8. Tables 4 and 8 show little difference between LQR and LQG controllers. Therefore, for limited number of measurements and reasonably small noises, LQG controller satisfying various constraints can be designed by adding Kalman filter to the LQR controller designed by the proposed optimization.

The order of LQG controller is the same as that of the design plant. To prevent time delay caused by computational burden, the controller order reduction is carried out using MATLAB command 'balmr' in Robust Control Toolbox (Chiang and Safonov 1997). This method is based on the balanced realization whose controllability grammian and observability grammian are same (Burl 1999). For constraint sets A, B and C, the order of original controller, 25 , can be reduced to 19,15 
Table 8 Peak responses of LQG controllers

\begin{tabular}{ccccccc}
\hline \hline Controller & Constraint & $\begin{array}{c}2^{\text {nd }} \\
\text { inter-story } \\
\text { drift }(\mathrm{cm})\end{array}$ & $\begin{array}{c}10^{\text {th }} \text { floor absolute } \\
\text { acceleration } \\
(\mathrm{g})\end{array}$ & $\begin{array}{c}\text { Stroke } \\
(\mathrm{m})\end{array}$ & $\begin{array}{c}\text { Control } \\
\text { force } \\
(\mathrm{kN})\end{array}$ & $\begin{array}{c}\text { Control } \\
\text { signal } \\
(\mathrm{V})\end{array}$ \\
\hline \multicolumn{2}{c}{ No control } & 3.67 & 0.30 & $\cdot$ & $\cdot$ & $\cdot$ \\
\hline \multicolumn{2}{c}{ Passive control } & 2.70 & 0.26 & 1.41 & $\cdot$ & $\cdot$ \\
\hline \multirow{2}{*}{ Full } & $\mathrm{A}$ & 2.01 & 0.18 & 1.51 & 10.21 & 33.2 \\
order & $\mathrm{B}$ & 2.00 & 0.20 & 1.75 & 5.83 & 17.4 \\
LQG & $\mathrm{C}$ & 2.00 & 0.20 & 1.94 & 5.62 & 15.9 \\
\hline \multirow{2}{*}{$\begin{array}{c}\text { Reduced } \\
\text { LQG }\end{array}$} & $\mathrm{A}$ & 2.00 & 0.18 & 1.51 & 10.23 & 33.3 \\
& $\mathrm{~B}$ & 2.00 & 0.20 & 1.75 & 5.88 & 17.7 \\
\hline
\end{tabular}

and 15, respectively. The peak responses with reduced LQG in Table 8 show insignificant performance deterioration compared to those of full order LQG.

\subsection{Verification of failure probability}

Since the failure probability presented in Eq. (13) is an approximation, simulations using 1,000 earthquake samples are performed to verify assumptions used in the proposed optimization method. Sample ground accelerations are generated by filtering white noises multiplied by the non-stationary envelope function through Kanai-Tajimi filter. Table 9, where the simulation result is presented, indicates that most failure probabilities of the activated constraints (distinguished by shade) are a little smaller than $5 \%$, which is the target value in the optimization. The reason is that structural responses do not reach to steady state at $t_{1}=20 \mathrm{sec}$., the beginning of stationary response assumed for the calculation of failure probability, due to the initial non-stationarity of the earthquake excitation. For the future study, a design method addressing this effect needs to be developed for more accurate estimation of failure probability.

Table 9 Failure probability obtained from 1000 simulations

\begin{tabular}{ccccccc}
\hline \hline Constraint & Controller & $\begin{array}{c}2^{\text {nd }} \\
\text { inter-story } \\
\text { drift }(\mathrm{cm})\end{array}$ & $\begin{array}{c}10^{\text {th }} \text { floor absolute } \\
\text { acceleration } \\
(\mathrm{g})\end{array}$ & $\begin{array}{c}\text { Stroke } \\
(\mathrm{m})\end{array}$ & $\begin{array}{c}\text { Control } \\
\text { force } \\
(\mathrm{kN})\end{array}$ & $\begin{array}{c}\text { Control } \\
\text { signal } \\
(\mathrm{V})\end{array}$ \\
\hline \multirow{4}{*}{ A } & LQR ( II $)$ & 4.1 & 0.9 & 3.9 & 4.6 & 4.1 \\
& LQG & 4.1 & 0.8 & 3.9 & 4.6 & 3.1 \\
& Reduced LQG & 4.2 & 0.8 & 4.0 & 5.0 & 3.1 \\
\hline \multirow{3}{*}{ B } & LQR ( II ) & 3.7 & 4.0 & 3.4 & 3.5 & 4.4 \\
& LQG & 3.6 & 4.1 & 3.5 & 3.7 & 4.1 \\
Ceduced LQG & 3.6 & 4.2 & 3.6 & 3.7 & 4.1 \\
\hline \multirow{4}{*}{ C } & LQR ( II ) & 4.7 & 4.2 & 1.5 & 5.4 & 3.7 \\
& Led & 4.6 & 4.2 & 1.6 & 5.3 & 5.0 \\
\hline
\end{tabular}




\section{Conclusions}

The present study proposes an optimal controller design method for the minimum control force with constraints on the failure probability of peak response. LQR is selected as control algorithm to keep closed-loop system stable easily during optimization procedure. Gradients of objective function and inequality constraints are derived to make use of general gradient based optimization algorithms.

Numerical analysis shows that the solution is not unique for various initial weightings. But different controllers, excluding a few exceptional results, show similar performance in terms of the peak control force and responses. The effects of stroke limitation and actuator cut-off frequency on control performance are investigated. It is observed that the strict constraint on stroke increases the peak control force due to the tendency of controlling higher modes. Also, the reduction of actuator cut-off frequency tends to increase the control signal rather than control force.

Performance degradation due to the output feedback using Kalman filter appended to the optimized LQR controller is found to be negligible. Further, the additional performance degradation due to the controller order reduction for LQG is not significant. Therefore, the proposed optimization of LQR can be used to design a low order output feedback controller for a structure with limited number of measured responses. Statistics of simulation results for 1,000 artificial earthquakes shows that failure probabilities of optimized control system are sufficiently accurate.

\section{Acknowledgements}

The work presented in this paper was partially supported by Research Fund of the National Research Laboratory Program (Project No. M1-0203-00-0068) from the Ministry of Science and Technology in Korea. The authors also gratefully acknowledge the support of this research by the Smart Infra-Structure Technology Center (SISTeC) (Project No. R11-2022-101-03004-0(2002)).

\section{References}

Anderson, B.D.O. and Moore, J.B. (1989), Optimal Control, Prentice Hall, Englewood Cliffs, NJ.

Ayorinde, E.O. and Warburton, G.B. (1980), "Minimizing structural vibrations with absorbers", Earthq. Eng. Struct. Dyn., 8, 219-236.

Belegundu, A.D. and Chandrupatla, T.R. (1999), Optimization Concepts and Applications in Engineering, Prentice Hall, NJ.

Burl, J.B. (1999), Linear Optimal Control, Addison-Wesley, CA.

Chiang, R.Y. and Safonov, M.G. (1997), Robust Control Toolbox, The Math Works, Inc.

Coleman, T., Branch, M.A. and Grace, A. (1999), Optimization Toolbox for Use with MATLAB, The Math Works, Inc.

Housner, G.W., Bergman, L.A., Caughey, T.K., Chassiakos, A.G., Claus, R.O., Masri, S.F., Skelton, R.E., Soong, T.T., Spencer, B.F. Jr. and Yao, J.T.P. (1997), "Special issue structural control: past, present, and future", J. Eng. Mech., ASCE, 123(9), 897-971.

Hsieh, C., Skelton, R.E. and Damra, F.M. (1989), "Minimum energy controllers with inequality constraints on output variances", Optimal Control Applications \& Methods, 10, 347-366.

Jennings, P.C., Housner, G.W. and Tsai, N.C. (1968), "Simulated earthquake motions", Rept. Earthquake Eng. Res. Lab., California Institute of Technology. 
Khargonekar, P.P. and Rotea, M.A. (1991), "Multiple objective optimal control of linear systems: The quadratic norm case", IEEE Transactions on Automatic Control, 36(1), 14-24.

Lin, Y.K. and Cai, G.Q. (1995), Probabilistic Structural Dynamics, McGraw-Hill, NY.

Lutes, L.D. and Sarkani, S. (1997), Stochastic Analysis of Structural and Mechanical Vibrations, Prentice Hall, NJ.

May, B.S. and Beck, J.L. (1998), "Probabilistic control for the active mass driver benchmark structural model", Earthq. Eng. Struct. Dyn., 27, 1331-1346.

Rotea, M.A. (1993), “The generalized $\mathrm{H}_{2}$ control problem”, Automatic, 29(2), 373-385.

Soong, T.T. (1990), Active Structural Control: Theory and Practice, Longman Wiley, London.

Spencer, B.F. Jr., Kaspari, D.C. and Sain, M.K. (1994a), "Structural control design: a reliability-based approach", Proc. of the American Control Conf., IEEE, 1062-1066.

Spencer, B.F. Jr., Suhardjo, J. and Sain, M.K. (1994b), "Frequency domain optimal control strategies for seismic protection", J. Eng. Mech., ASCE, 120(1), 135-158.

Toivonen, H.T. and Mäkilä, P.M. (1989), "Computer-aided design procedure for multi-objective LQG control problems", International Journal of Control, 49(2), 655-666.

Yang, J.N., Wu, J.C., Reinhorn, A.M., Riley, M., Schmitendorf, W.E. and Jabbari, F. (1996), "Experimental verification of $\mathrm{H}_{\infty}$ and sliding mode control for seismically excited buildings", J. Struct. Eng., 122(1), 69-75.

Zhu, G. and Skelton, R. (1991), "Mixed $\mathrm{L}_{2}$ and $\mathrm{L}_{\infty}$ problems by weight selection in quadratic optimal control", International Journal of Control, 53(5), 1161-1176.

Zhu, G., Rotea, M.A. and Skelton, R. (1997), "A convergent algorithm for the output covariance constraint control problem”, SIAM Journal of Control and Optimization, 35(1), 341-361. 